\title{
Random Resonators and Prelocalized Modes in Disordered Dielectric Films.
}

\author{
V. M. Apalkov and M. E. Raikh \\ Department of Physics, University of Utah, Salt Lake City, UT 84112, USA \\ B. Shapiro \\ Department of Physics, Technion-Israel Institute of Technology, Haifa 32000, Israel
}

\begin{abstract}
Areal density of disorder-induced resonators with a high quality factor, $Q \gg 1$, in a film with fluctuating refraction index is calculated theoretically. We demonstrate that for a given $k l>1$, where $k$ is the light wave vector, and $l$ is the transport mean free path, when on average the light propagation is diffusive, the likelihood for finding a random resonator increases dramatically with increasing the correlation radius of the disorder. Parameters of most probable resonators as functions of $Q$ and $k l$ are found.
\end{abstract}

PACS numbers: 42.25.Dd, 42.60.Da, 73.20.Fz

Introduction. Recent discovery of the coherent lasing from various disordered materials adds a new dimension to the conventional physics of light propagation in multiply scattering media. In the experimental works 11 《 it was demonstrated that, above a certain excitation level, the emission spectra of $Z n O$ powders [1,2], conjugated polymer films [3] and dye-infiltrated opals [4] exhibit a sequence of extremely narrow peaks, their widths being limited by the spectrometer resolution. Until recently, this finite resolution left some room for doubt as to whether the observed peaks indicated true lasing [5]. However, the latest experiments on photon statistics [6,7] have unambiguously established the coherence of the emitted light, thus proving conclusively that the underlying mechanism of random lasing in Refs. [1 involves the amplitude (coherent) rather than power (incoherent) feedback. (The latter is known to occur via the diffusion process of the light intensity, as proposed long ago by Letokhov [8] and observed first by Lawandy [9] and later in other numerous experiments.) The origin of coherent feedback, responsible for the lasing observed in Refs. [1 1 , is a subject of controversy and debate. Such a feedback would naturally emerge if the light were localized. However, the coherent backscattering measurements [1,10-14] carried out in parallel with the analysis of the emission spectra rule out this possibility. Firstly, the values of $k l$ extracted from these measurements turn out rather large. Secondly, the onset of the Anderson localization manifests itself in the rounding of the top of backscattering cone [15 17. No such rounding was observed in the experiments 11.10 14].

In the early work of Cao et. al. 11,10,11], it was argued that, even in diffusive regime of light propagation, a photon, scattered off a certain sequence of impurities (grains of, roughly, 100 $\mathrm{nm}$ size) can move on a closed loop, which thereby serves as a laser resonator. The picture of such ring cavities put forward in Refs. [1, 10,11] was based on the experimental evidence [18] that the recurrent scattering processes contribute to backscattering albedo. However, since in each scattering act most of the energy gets scattered out of the loop, an unrealistically high gain would be required to achieve the lasing threshold condition for such a loop. This point was particularly emphasized by van Soest [14], who also argued that "impurity loops" are likely to generate a broad frequency spectrum rather than isolated resonances.

Certainly the picture of random cavities representing a certain spatial arrangement of isolated scatterers is too naive. This, however, does not rule out the entire concept of disorder-induced resonators. Although sparse, the disorder configurations that trap the light for long enough time can occur in a sample of a large enough size, and a single such configuration is already sufficient for lasing to occur. Therefore, under the condition $k l \gg 1$, which implies that overall scattering is weak, the conclusion about the relevance of random cavities can be drawn only upon the quantitative calculation of their likelihood. This is the subject of the present paper.

We start with a remark that the issue of random resonators for light waves has its counterpart in the electronic transport. In particular, the modes with anomalously low losses are analogous to the so called prelocalized electronic states in diffusive conductors that are responsible for the long-time asymptotics of the current relaxation. Theoretical study of these states was launched more than a decade ago (see Ref. [19) and later renewed in Ref. 20. The results obtained to date are summarized in the review [21]. Following this analogy we dub the modes of random resonators with anomalously high quality factor, $Q$, as prelocalized modes.

The principal outcome of our study is that for a given $k l \gg 1$, the probability of formation of a high- $Q$ random resonator depends crucially on the size of the scatterers, or, more precisely, on the correlation radius of the disorder, $R_{c}$. Similarly to the treatment in Refs. [2]11] we restrict our consideration to the two-dimensional case (a disordered film). Regarding the geometry of a random resonator, we adopt the idea proposed by Karpov in Ref. [22] for trapping the acoustic waves in three dimensions. According to Ref. [22], the fluctuations responsible for trapping are the toroidal inclusions with reduced sound velocity. Correspondingly, in two dimensions, a random resonator represents a 
ring-shape area (see Fig. 1a) within which the dielectric constant is enhanced by some small value $\epsilon_{1}$ (compared to the background value $\epsilon_{0}$ ). Then such a ring can be viewed as a waveguide that supports the modes of a whispering-gallery type. Due to the azimuthal symmetry, these modes are characterized by the radial index and the angular momentum, $m$. Denote by $\mathcal{N}_{m}(k l, Q)$ the areal density of resonators with quality factor $Q$ in the film with a transport mean free path $l$. Here $k=\epsilon_{0}^{1 / 2} k_{0}$, where $k_{0}=2 \pi / \lambda, \lambda$ stands for the wavelength in vacuum. Obviously, in the diffusive regime, $k l>1$, the density $\mathcal{N}_{m}(k l, Q)$ is exponentially small for $Q \gg 1$. In this domain $\mathcal{N}_{m}(k l, Q)$ can be presented as

$$
\mathcal{N}_{m}(k l, Q)=\mathcal{N}_{0} e^{-S_{m}(k l, Q)},
$$

where $\mathcal{N}_{0}$ is the prefactor. Now we can "quantify" the above statement about the dependence of the resonator likelihood on $R_{c}$

$$
\frac{S_{m}\left(k_{0} R_{c}>1\right)}{S_{m}\left(k_{0} R_{c} \ll 1\right)}=\frac{\Phi\left(\epsilon_{1}^{1 / 2} k_{0} R_{c}\right)}{\pi^{1 / 2}\left(\epsilon_{0}^{1 / 2} k_{0} R_{c}\right)^{3}} .
$$

The dimensionless single-parameter function $\Phi$ is shown in Fig. 2. It is seen from Eq. (2) that $S_{m}$ falls off rapidly with increasing $R_{c}$. In the domain $k_{0} R_{c}>1$ but $\epsilon_{1}^{1 / 2} k_{0} R_{c} \lesssim 1$ we can set $\Phi \approx 1$, so that we have $S_{m} \propto\left(k_{0} R_{c}\right)^{-3}$. For larger $R_{c}$, when $\epsilon_{1}^{1 / 2} k_{0} R_{c} \gg 1$, the function $\Phi$ behaves as $\Phi(u) \propto u$. In this domain $S_{m}$ decreases slower with $R_{c}: S_{m} \propto\left(k_{0} R_{c}\right)^{-2}$. We emphasize again that Eq. (2) applies for a given $k l$ value, so that the decrease of $S_{m}$ with $R_{c}$ leaves the backscattering cone unchanged. Below we will demonstrate with rigorous calculations that the value of $S_{m}\left(k_{0} R_{c} \ll 1\right)$ is really large

$$
S_{m}\left(k_{0} R_{c} \ll 1\right)=2\left(\frac{\pi^{3}}{3}\right)^{1 / 2} k l|\ln Q|,
$$

which makes formation of random resonators with $Q \gg 1$ practically impossible for point-like scatterers. On the contrary, for $k_{0} R_{c} \gtrsim 2$ ring-like fluctuations of the dielectric constant (see Fig. $1 \mathrm{~b}$ ) make realistic candidates for high- $Q$ random resonators.

Derivation of Eq. (国). Denote by $P$ the probability of fluctuation of dielectric constant, $\delta \epsilon(\boldsymbol{r})$. Assuming the fluctuations to be Gaussian with r.m.s. $\Delta$, we have

$$
\ln P=-\frac{1}{2 \Delta^{2}} \iint d \boldsymbol{r} d \boldsymbol{r}^{\prime} \delta \epsilon(\boldsymbol{r}) \delta \epsilon\left(\boldsymbol{r}^{\prime}\right) \kappa\left(\boldsymbol{r}-\boldsymbol{r}^{\prime}\right),
$$

where the kernel $\kappa(\boldsymbol{r})$ is related in the usual way, $\int d \boldsymbol{r}^{\prime} \kappa\left(\boldsymbol{r}-\boldsymbol{r}^{\prime}\right) K\left(\boldsymbol{r}^{\prime}-\boldsymbol{r}_{1}\right)=\delta\left(\boldsymbol{r}-\boldsymbol{r}_{1}\right)$, to the correlation function, $K(\boldsymbol{r})$, defined as

$$
\left\langle\delta \epsilon(\boldsymbol{r}) \delta \epsilon\left(\boldsymbol{r}^{\prime}\right)\right\rangle=\Delta^{2} K\left(\boldsymbol{r}-\boldsymbol{r}^{\prime}\right) .
$$

We search for fluctuations of a type shown in Fig. 1, i.e., $\delta \epsilon$ is azimuthally symmetric (depends only on the radius, $\rho$ ) and is non-zero within the relatively narrow ring of width $w \ll \rho_{0}$, where $\rho_{0}$ is the radius corresponding to the middle of the ring (see Fig. 1a). For such a fluctuation the wave equation for field distribution corresponding to the angular momentum, $m$, reduces to

$$
\hat{L} \chi_{m}=\frac{d^{2} \chi_{m}}{d x^{2}}+\delta \epsilon k_{0}^{2} \chi_{m}=\epsilon_{1} k_{0}^{2} \chi_{m}
$$

where $x=\rho-\rho_{0}$. The "eigenvalue" in the r.h.s. of Eq. (6) is defined as $\epsilon_{1}=\left(\frac{m}{k_{0} \rho_{0}}\right)^{2}-\epsilon_{0}$. It is independent of $x$ by virtue of the condition $\rho_{0} \gg w$. The next step is to find the "most probable" distribution $\delta \epsilon(x)$ for a given $\epsilon_{1}$. This is done within the standard optimal fluctuation approach [23.24], which prescribes minimization of auxiliary functional $\Psi\{\delta \epsilon\}=\ln P-\lambda\left(\chi_{m} \hat{L} \chi_{m}\right)$, where $\lambda$ is the Lagrange multiplier. This yields

$$
\delta \epsilon(x)=\int d x_{1} K_{0}\left(x-x_{1}\right) \chi_{m}^{2}\left(x_{1}\right),
$$

where we have set $\lambda=2 \pi \rho_{0} / \Delta^{2} k_{0}^{2}$. The above expression reminds the standard result of optimal fluctuation approach for a correlated random potential [23]. The only difference is that, due to the angular integration in Eq. (卌), the kernel in Eq. (耳) is given by a function $K_{0}$, which is related to the correlator, $K(\boldsymbol{r})$, as 
$K_{0}\left(x_{1}-x_{2}\right)=\int_{-\infty}^{\infty} d y K\left(\sqrt{\left(x_{1}-x_{2}\right)^{2}+y^{2}}\right)$. A natural $x$-scale for the eigenmode $\chi_{m}$ is $\left(\epsilon_{1}^{1 / 2} k_{0}\right)^{-1}$. Thus, we present the outcome of the optimal fluctuation approach in terms of a dimensionless variable $z=\epsilon_{1}^{1 / 2} k_{0} x$.

$$
S_{m}=\frac{\pi \rho_{0}}{\epsilon_{1} k_{0}^{2} \Delta^{2}} \iint d z_{1} d z_{2} \chi_{m}^{2}\left(z_{1}\right) \chi_{m}^{2}\left(z_{2}\right) K_{0}\left(z_{1}-z_{2}\right) .
$$

The dimensionless equation for the function $\chi_{m}$ reads

$$
\frac{d^{2} \chi_{m}(z)}{d z^{2}}+\frac{\chi_{m}(z)}{\epsilon_{1}^{3 / 2} k_{0}} \int d z_{1} K_{0}\left(z-z_{1}\right) \chi_{m}^{2}\left(z_{1}\right)=\chi_{m}(z) .
$$

To proceed further we have to specify the correlator, $K$. We have chosen the Gaussian form $K(\rho)=\exp \left(-\rho^{2} / R_{c}^{2}\right)$. The form of the function $\chi_{m}=A\left(R_{c}\right) \exp \left[-\gamma\left(R_{c}\right) z^{2}\right]$ allows to cover the entire range of correlation radii, from "white noise" $\left(R_{c} \rightarrow 0\right)$ to the limit of a smooth disorder $\left(k_{0} R_{c} \gg 1\right)$. Indeed, for large $R_{c}$ this form becomes exact. In the opposite limit, $R_{c} \rightarrow 0$, using the above trial function instead of exact solution $\chi_{m} \propto 1 / \cosh (z)$ leads to the overestimate of $S_{m}$ by a factor $(\pi / 3)^{1 / 2} \approx 1.023$. The parameter $A\left(R_{c}\right)$ and $\gamma\left(R_{c}\right)$ of the trial function can be found analytically. Evaluation of Eq. (8) reduces to the Gaussian integration which for a given $m \approx \epsilon^{1 / 2} k_{0} \rho_{0}$ yields

$$
S_{m}=2^{4} 3^{-3 / 2} \pi^{1 / 2} m\left(\frac{\epsilon_{1}^{3}}{\epsilon_{0}}\right)^{1 / 2} \frac{\Phi\left(\epsilon_{1}^{1 / 2} k_{0} R_{c}\right)}{\left(\Delta k_{0} R_{c}\right)^{2}} .
$$

The analytical expression for the function $\Phi(u)$ introduced above in Eq. (2), and shown in Fig. 2, is the following

$$
\Phi(u)=\frac{3^{3 / 2}}{16}\left(\frac{5+\sqrt{9+16 u^{2}}}{3+\sqrt{9+16 u^{2}}}\right)^{2}\left[u^{2}+\frac{1}{2}\left(3+\sqrt{9+16 u^{2}}\right)\right]^{1 / 2} .
$$

Recall that we are interested in the density of random resonators at a given value of $k l$. The remaining task is to express the transport mean free path in terms of $\Delta$ and $R_{c}$. With $K(\rho)=\exp \left(-\rho^{2} / R_{c}^{2}\right)$ the expression simplifies in two limits

$$
\begin{aligned}
& k l=\frac{4 \epsilon_{0}}{\pi\left(k_{0} R_{c} \Delta\right)^{2}} \text { for } k_{0} R_{c} \ll 1 ; \\
& k l=\frac{4 \epsilon_{0}^{5 / 2} k_{0} R_{c}}{\pi^{1 / 2} \Delta^{2}} \text { for } \quad k_{0} R_{c} \gg 1 .
\end{aligned}
$$

Note, that Eqs. (12a), (12b) can be cast into the conventional form $l \propto 1 / n \sigma$, where $n$ is the concentration of scatterers and $\sigma$ is the transport cross section. In particular, Eq. (12a) follows from the two-dimensional version $\left(\sigma \propto R_{c}^{4} k_{0}^{3}\right)$ of the Rayleigh scattering formula for $\sigma$. Combining Eq. (10) and Eqs. (12a), (12b), we arrive at Eq. (22). In the limit $\epsilon_{1}^{1 / 2} k_{0} R_{c} \gg 1$ Eq. (10) has a simple interpretation. Namely, in this limit $S_{m}$ can be rewritten as $S_{m} \sim\left(\epsilon_{1} / \Delta\right)^{2}\left(\rho_{0} / R_{c}\right)$. The first factor comes from the Gaussian probability to have a fluctuation $\epsilon_{1}$ within a square of an area $\sim R_{c}^{2}$. The second factor accounts for the number of squares needed to cover the ring area $A_{m} \sim \rho_{0} R_{c}$.

$Q$-factor. It is obvious that at large distances, $\rho \gg \rho_{0}$, the behavior of $\chi_{m}$ is oscillatory, $\chi_{m} \propto \exp \left(i \epsilon_{0}^{1 / 2} k_{0} \rho\right)$, manifesting that the waveguided mode of a ring, being prelocalized, has a finite lifetime. In other words, the eigenvalue, $\epsilon_{1}$, in Eq. (6) has an imaginary part, $\tilde{\epsilon}_{1}$, due to evanescent leakage. The quality factor is inversely proportional to $\tilde{\epsilon}_{1}$, namely, $Q=\epsilon_{0} / \tilde{\epsilon}_{1}$. The leading contribution to $\tilde{\epsilon}_{1}$ comes from the region of a width, $d$, adjacent to the waveguide (see Fig. 1a). To find $d$ we have to take into account that the r.h.s in Eq. (6) does in fact depend on $x$. This is because the precise form of the r.h.s is not $\left(\frac{m}{\rho_{0}}\right)^{2}-\epsilon_{0} k_{0}^{2}$, but rather $\left(\frac{m}{\rho_{0}+x}\right)^{2}-\epsilon_{0} k_{0}^{2}$. In the region outside the waveguide, when $x \gg 1 / \epsilon_{1}^{1 / 2} k_{0}$ (but still $x \ll \rho_{0}$ ) Eq. (6) takes the form

$$
\frac{d^{2} \chi_{m}}{d x^{2}}=\epsilon_{1} k_{0}^{2}\left(1-\frac{x}{d}\right) \chi_{m}
$$

where the width of the decay region is given by $d=\epsilon_{1} k_{0}^{2} \rho_{0}^{3} / 2 m^{2}$. Equation (13) is of Airy-type. Semiclassical calculations with exponential accuracy yields for the rate of evanescent leakage $\tilde{\epsilon}_{1} \propto \exp \left[-(2 m / 3)\left(\epsilon_{1} / \epsilon_{0}\right)^{3 / 2}\right]$, and hence 


$$
\ln Q=\frac{2 m}{3}\left(\frac{\epsilon_{1}}{\epsilon_{0}}\right)^{3 / 2}
$$

Substituting $\epsilon_{1}$ from Eq. (14) and $\Delta$ from Eq. (12a) into Eq. (10) we arrive at Eq. (3). The above derivation relied on the assumptions $\rho_{0} \gg d$ and $d \gg w$. These assumptions are justified within the following domain of the quality factors $m \gg \ln Q \gg \max \left\{1, \epsilon_{0}^{3 / 4}\left(k_{0} R_{c}\right)^{3 / 2} m^{-1 / 2}\right\}$.

Discussion. Equation (3) quantifies the effectiveness of trapping of light in a random medium with point-like scatterers. It follows from Eq. (3) that the likelihood of high- $Q$ cavity is really small. Indeed, even for rather strong disorder, $k l=5$, the exponent, $S_{m}$, in the probability of having a cavity with a quality factor $Q=50$ is close to $S_{m}=120$. We emphasize that in two dimensional case under consideration, this exponent does not depend on $m$ and, thus, on the cavity radius $\rho_{0}=m / \epsilon_{0}^{1 / 2} k_{0}$. To estimate the degree to which finite size of scatterers $\left(\sim R_{c}\right)$ improves the situation we choose $k_{0} R_{c} \approx 2$, which already corresponds to the limit $k_{0} R_{c} \gg 1$ in Eq. $(12 \mathrm{~b})$, but still allows to set $\Phi=1$. Then for $Q=50, k l=5$ we obtain $S_{m} \approx 1$.1, suggesting that the resonators with this $Q$ are quite frequent. In the latter estimate we have set $\epsilon_{0}=4$.

A natural question to address is how large a value of $Q$ can be achieved for a given $k l$ and $k_{0} R_{c}$. To address this question we inspect the argument $u=\epsilon_{1}^{1 / 2} k_{0} R_{c}$ of the function $\Phi$. With the use of Eq. (14) it can be presented in the form $u=\epsilon_{0}^{1 / 2} k_{0} R_{c}\left[\frac{3 \ln Q}{2 m}\right]^{1 / 3}$. Since $\Phi(u)$ increases monotonically (see Fig. 2), the latter expression suggests that $Q$ can be increased at the expense of larger $m$-values. In the example considered above, in order to keep $\Phi$ smaller than, say $1.5, m$ should be bigger than 50. However, due to slow dependence $u \propto m^{-1 / 3}$, we get rather small value $S_{m} \approx 3$ for $m$ as small as $m=15$. Certainly, the allowed values of $m$ are limited from above. This limitation originates from "vulnerability" of waveguiding to the fluctuations of the dielectric constant around optimal ring-like distribution. The dangerous fluctuations are those that enhance the evanescent leakage. Note, that these fluctuations do not affect the main exponent $S_{m}$ in the density of resonators. It is obvious that the bigger is the area $A_{m}=2 \pi \rho_{0} d \propto m^{4 / 3}$, responsible for evanescent leakage (see Fig. 1a), the harder it is to "protect" the waveguiding. Since the fluctuations $\delta \epsilon(\boldsymbol{r})$ have the spatial scale $R_{c}$, the probability that the waveguiding "survives" can be roughly estimated as $\exp \left(-A_{m} / R_{c}^{2}\right)$. The condition that the exponent $A_{m} / R_{c}^{2}$ does not dominate over the principal exponent $S_{m}$ can be rewritten as $m \lesssim\left(\epsilon_{0} k l\right)^{3 / 5}(\ln Q)^{2 / 5}$. For the example $k l=5$ and $Q=50$, addressed above, we get $m \lesssim 10$. Rigorous calculation of the "survival probability" is the problem of the same complexity as calculation of the prefactor in the functional integral [25].

Conclusion. In the present paper we provided a quantitative theory of random resonators that substantiates the intuitive image [1, 10] of a resonant cavity as a closed-loop trajectory of a light wave bouncing between the point-like scatterers. The intuitive picture in [1, 10] assumed that light can propagate along a loop of scatterers by simply being scattered from one scatterer to another. Such a picture, however, is unrealistic due to the scattering out of the loop 114. We have demonstrated that the scenario of light traveling along closed loops can be remedied. In our picture the "loops", i.e. the random resonators, can be envisaged as rings with dielectric constant larger than the average value. On a microscopic level these resonators correspond to certain arrangements of scatterers (grains). The main point, however, is that a resonator acts as a single entity: only the coherent multiple scattering of light by all the scatterers in the resonator can provide trapping. We also point out that correlations in the fluctuating part of the dielectric constant (due to finite grain size) highly facilitate trapping.

The effectiveness of light trapping is expressed by Eq. (2). This expression describes the statistics of the quality factors which determines the distribution of the threshold gain for random lasing. Our consideration pertains to the passive disordered films, in the sense, that we neglect the effect of gain on the spatial distribution of the light intensity [2, 11, 26, 27]. Random resonators considered in the present paper are sparse, so that there is no spatial overlap between the modes of different resonators, the situation opposite to that considered in Refs. [28,29]. We have also treated scatterers as frequency-independent fluctuations, $\delta \epsilon(\boldsymbol{r})$, of the dielectric constant. The entirely different scenario of the collective mode formation emerges for resonant scatterers [30].

Acknowledgments. Two of the authors MER and BS acknowledge the hospitality of the University of Köln and of the Institute for Theoretical Physics at UCSB where the parts of this work were completed. This research was supported in part by the National Science Foundation under Grant No. PHY99-07949.

[1] H. Cao, Y. G. Zhao, S. T. Ho, E. W. Seelig, Q. H. Wang, and R. P. H. Chang, Phys. Rev. Lett. 82, 2278 (1999), 
[2] H. Cao, J. Y. Xu, D. Z. Zhang, S.-H. Chang, S. T. Ho, E. W. Seelig, X. Liu, and R. P. H. Chang, Phys. Rev. Lett. 84, $5584(2000)$.

[3] S. V. Frolov, Z. V. Vardeny, K. Yoshino, A. Zakhidov, and R. H. Baughman, Phys. Rev. B 59, R5284 (1999).

[4] S. V. Frolov, Z. V. Vardeny, A. A. Zakhidov, and R. H. Baughman, Opt. Commun. 162, 241 (1999).

[5] D. Wiersma, Nature 406, 132 (2000).

[6] H. Cao, Y. Ling, J. Y. Xu, C. Q. Cao, and P. Kumar, Phys. Rev. Lett. 86, 4524 (2001).

[7] R. C. Polson, A. Chipouline, and Z. V. Vardeny, Adv. Mater. 13, 760 (2001).

[8] V. S. Letokhov, Zh. Éksp. Teor. Fiz. 53, 1442 (1967) [Sov. Phys. JETP 26, 835 (1968)].

[9] N. M. Lawandy, R. M. Balachandran, A. S. L. Gomes, and E. Sauvain, Nature (London) 368, 436 (1994).

[10] H. Cao, Y. G. Zhao, H. C. Ong, and R. P. H. Chang, Phys. Rev. B 59, 15107 (1999).

[11] H. Cao, J. Y. Xu, S.-H. Chang, and S. T. Ho, Phys. Rev. E 61, 1985 (2000).

[12] G. van Soest, F. J. Poelwijk, R. Sprik, and A. Lagendijk, Phys. Rev. Lett. 86, 1522 (2001).

[13] R. C. Polson, M. E. Raikh, and Z. V. Vardeny, ArXiv: cond-mat 0105360.

[14] G. van Soest, Ph.D. thesis, Universiteit van Amsterdam, The Netherlands, 2001 (unpublished).

[15] F. J. P. Schuurmans, M. Megens. D. Vanmaekelbergh, and A. Lagendijk, Phys. Rev. Lett. 83, 2183 (1999).

[16] R. Berkovits and M. Kaveh, Phys. Rev. B 36, 9322 (1987).

[17] I. Edrei and M. J. Stephen, Phys. Rev. B 42, 110 (1990).

[18] D. S. Wiersma, M. P. van Albada, B. A. van Tiggelen, and A. Lagendijk, Phys. Rev. Lett. 75, 1739 (1995).

[19] B. L. Altshuler, V. E. Kravtsov, I. V. Lerner, in Mesoscopic Phenomena in Solids, eds. B. L. Altshuler, P. A. Lee, and R. A. Webb (North Holland, Amsterdam, 1991).

[20] B. A. Muzykantskii and D. E. Khmelnitskii, Phys. Rev. B 51, 5480 (1995).

[21] A. D. Mirlin, Phys. Rep. 326, 259 (2000).

[22] V. G. Karpov, Phys. Rev. B 48, 12539 (1993).

[23] B. I. Halperin and M. Lax, Phys. Rev. 148, 722 (1966).

[24] J. Zittartz and J. S. Langer, Phys. Rev. 148, 741 (1966).

[25] E. Brezin and G. Parisi, J. Phys. C 13 L307 (1980).

[26] X. Jiang and C. M. Soukoulis, Phys. Rev. Lett. 85, 70 (2000).

[27] X. Jiang and C. M. Soukoulis, ArXiv cond-mat/0103042.

[28] C. W. J. Beenakker, J. C. J. Paasschens, and P. W. Brouwer, Phys. Rev. Lett. 76, 1368 (1996).

[29] T. Sh. Misirpashaev and C. W. J. Beenakker, Phys. Rev. A 57, 2041 (1998).

[30] T. V. Shahbazyan, M. E. Raikh, and Z. V. Vardeny Phys. Rev. B 61, 13266 (2000). 


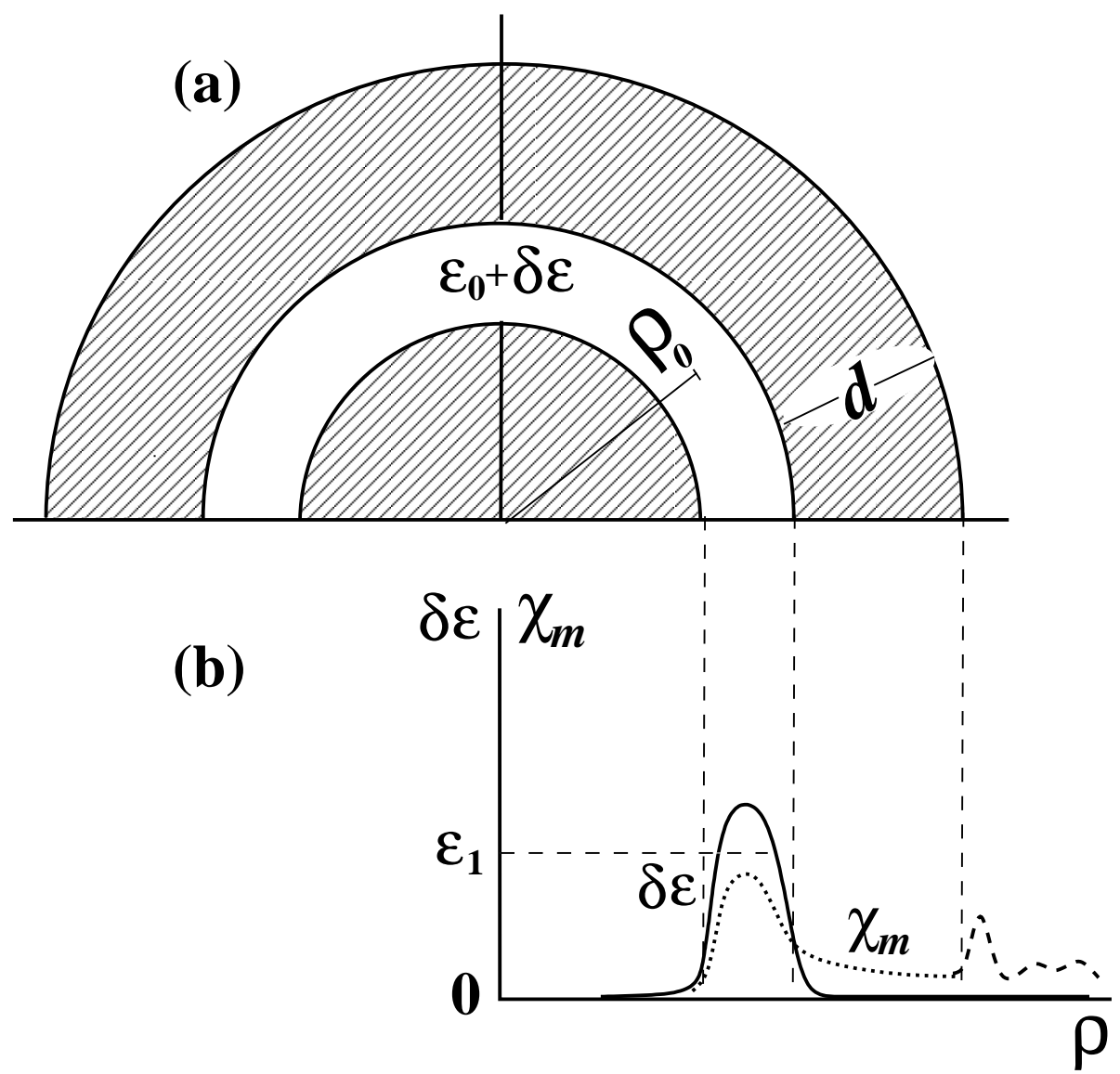

FIG. 1. (a) The structure of a two-dimensional resonator is illustrated schematically; only a half of the ring-shaped waveguide (blank region) is shown. (b) Optimal fluctuation of the dielectric constant, $\delta \epsilon(\rho)$ (solid line), and the corresponding field distribution (dotted line) are shown. Dashed line outside the shaded region of a width, $d$, illustrates the evanescent leakage.

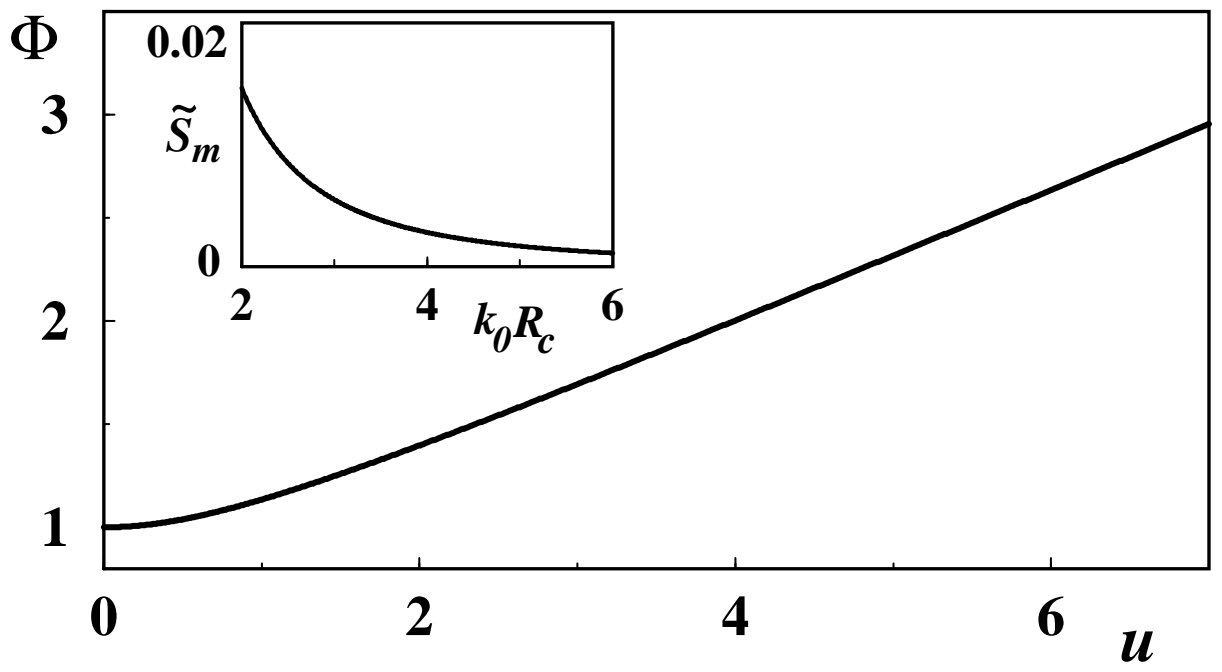

FIG. 2. Dimensionless function $\Phi(u)$ defined in Eq. (11) is plotted. Inset: Normalized modulus of the log-density of random resonators, $\tilde{S}_{m}=S_{m}\left(k_{0} R_{c}\right) / S_{m}(0)$, calculated from Eq. (2) for $\epsilon_{0}=4, Q=50$, and $m=15$ is plotted versus the dimensionless correlation radius, $k_{0} R_{c}$. 